\title{
Propiedades Psicométricas del Inventario de Estrategias de Autorregulación en Estudiantes de Pedagogía Chilenos
}

\author{
Carolina Zambrano ${ }^{1 *}$, Darío Rojas ${ }^{1}$, Alejandro Díaz ${ }^{2}$ y Pedro Salcedo ${ }^{1}$ \\ 1 Departamento de Metodología de Investigación e Informática Educacional, Facultad de Educación \\ Universidad de Concepción, Concepción, Chile (e-mail:carolinazambrano@gmail.com). \\ 2 Departamento de Psicología, Facultad de Ciencias Sociales, Universidad de Concepción, Concepción, \\ Chile. (e-mail: adiazm@udec.cl).
}

${ }^{*}$ Autor a quien debe ser enviada la correspondencia

Recibido Ene. 15, 2018; Aceptado Mar. 21, 2018; Versión final Abr. 13, 2018, Publicado Oct. 2018

\begin{abstract}
Resumen
El objetivo de la investigación es estudiar las propiedades psicométricas de la Escala Inventario de Estrategias de Autorregulación, en una población de estudiantes universitarios chilenos de programas de pregrado en pedagogía. Para dar respuesta al objetivo se realizó un estudio instrumental que incluye el análisis de las propiedades psicométricas de la escala a través de un análisis factorial exploratorio y análisis factorial confirmatorio en una muestra de 382 estudiantes. Los resultados del análisis factorial confirmatorio revelaron índices de ajuste adecuados para el modelo de cuatro factores. Estos son, organización del entorno, organización de la tarea, búsqueda de información y hábitos inadecuados de regulación. Se concluye que el Inventario de Estrategias de Autorregulación es un instrumento válido y fiable para ser utilizado en esta población.
\end{abstract}

\section{Psychometric Properties of the Inventory of Self-regulation Strategies in Chilean Pedagogy Students}

\begin{abstract}
The objective of the research is to study the psychometric properties of the Inventory Scale of Self-Regulation Strategies, in a population of Chilean university students of undergraduate programs in pedagogy. To reach this objective, an instrumental study that included the analysis of the psychometric properties of the scale through an exploratory factorial analysis and confirmatory factor analysis in a sample of 382 students was carried out. The results of the confirmatory factor analysis revealed adequate adjustment indexes for the four factors model. These are, organization of the environment, organization of the task, search for information and inadequate regulatory habits. It is concluded that the Inventory of Self-Regulation Strategies is a valid and reliable instrument to be used in this population.
\end{abstract}

Keywords: self-regulation learning, strategies of self-regulation, confirmatory factor analysis, psychometry 


\section{INTRODUCCIÓN}

El concepto autorregulación del aprendizaje (ARA) ha sido definido por varios autores entre los que destacan Zimmerman (2000), Pintrich (1990), Boekaerts (1995), Winne (1996), entre otros. Al respecto parece existir consenso entre las distintas definiciones que sugieren que ARA es el proceso por el cual un estudiante puede manejar sus cogniciones, comportamientos y emociones para maximizar su capacidad de aprendizaje. También, en los últimos años la evidencia respalda que ARA es un factor predictor del logro académico (Boekaerts et. al, 2005; Sitzmann y Ely, 2011; Mega et. al, 2014; Díaz y Pérez, 2013; Hernández y Camargo, 2017b). También, se ha señalado que ARA es un proceso complejo, tanto estructural como procesualmente (Zimmerman, 2000). Por la complejidad de este constructo se hace necesario especificar qué variable y/o proceso es el que se desea medir para desarrollar investigaciones con instrumentos que midan los factores 0 dimensiones de ARA de forma valida y fiable (Streiner et. al, 2015; Batista-Foguet et. al, 2004). En este sentido, ARA puede ser medido en una de las áreas o dimensiones de la autorregulación versus un planteamiento holístico y multidimensional. Por lo tanto, es decisión del investigador aplicar instrumentos válidos de acuerdo con un enfoque holístico o uno específico de ARA. Por ejemplo, el instrumento SRSI-SR adaptado (Hernández y Camargo, 2017) mide la dimensión estrategias de autorregulación y el instrumento Motivated Strategies for Learning Questionnaire (MSLQ) de Pintrich et. al (1991) mide ARA de forma multidimensional con cuatro dimensiones.

En el contexto de estrategias de autorregulación, actualmente varias investigaciones han constatado que esta dimensión representa un componente clave en los procesos de aprendizaje (Salmerón et. al., 2011, entre otras). Conceptualmente las estrategias de autorregulación se refieren al grado de control que ejerce el estudiante sobre los procesos, metas y contenidos académicos (Gutiérrez-Braojos et. al., 2014). Por ello, el uso de estas estrategias "provee a los estudiantes un mayor control sobre su propio proceso de aprendizaje en la adquisición de conocimientos y destrezas" (Elvira-Valdés y Pujol, 2012, p.368). Los hallazgos de los estudios señalan que las estrategias de autorregulación se relacionan positivamente con el rendimiento académico (Salmerón et al., 2011; Cleary y Platten, 2013; Gutiérrez-Braojos et. al., 2014; Broadbent y Poon, 2015). Por el contrario, cuando existe carencia de estrategias de autorregulación el estudiante presenta dificultades para advertir qué es lo que se le pide, le resulta difícil guiarse por los objetivos de una materia, no comprende claramente las instrucciones que se le presentan, o no sabe a quién pedir ayuda ni cómo buscarla (Salmerón et al., 2011). También, se ha evidenciado que las estrategias de autorregulación afectan positivamente los resultados de aprendizaje, ayudando a los estudiantes a adquirir y retener conocimiento de una manera estructurada y metódica (Broadbent y Poon, 2015). En suma, las estrategias de autorregulación son necesarias para el estudiante universitario porque quienes las aplican pueden llegar a ser capaces de planificar, monitorear y evaluar el proceso en la consecución de objetivos de aprendizaje (Gutiérrez-Braojos et. al., 2014).

Por lo anterior, se hace necesario contar con diagnósticos de las capacidades relacionadas con el uso de estrategias de autorregulación en estudiantes universitarios que ingresan a primer año. El diagnóstico adquiere importancia si se considera que en Chile existen altas tasas de deserción en estudiantes universitarios. En términos generales la deserción se produce porque estos no logran adecuarse a las exigencias de la vida universitaria (Cazan, 2012). En particular, las cifras en Chile muestran que el $25 \%$ de los estudiantes deserta al primer año de acuerdo con datos del Informe de Retención de 1er año de pregrado cohortes 2011-2015 (SIES, 2016). Por tanto, es necesario contar con instrumentos de medición válidos y fiables de la variable estrategias de autorregulación, para realizar diagnósticos que posteriormente podrían dar origen a una mejor planificación de programas intracurriculares de intervención y/o fomento de esta capacidad que se puede enseñar (Boekaerts et. al., 2005). En este sentido, la Escala Inventario de Estrategias de Autorregulación (SRSI-SR por sus siglas en inglés) desarrollada por Cleary (2006) para estudiantes de educación secundaria, consta de 28 ítems en inglés y mide estrategias de ARA en el ámbito de las ciencias para nivel de secundaria. Dado que el instrumento SRSI-SR fue diseñado para estudiantes de secundaria Hernández y Camargo (2017a) lo adaptan en tres aspectos principales que son: "idioma, población objetivo y orientación de la evaluación" (Hernández y Camargo, 2017a, p.14).

Es decir, la adaptación de Hernández y Camargo (2017a) es a idioma español, población universitaria y para ser usado en cualquier asignatura. Así, se crea el instrumento SRSI-SR adaptado que está compuesto por 18 ítems y cuatro subescalas que son: 1) Organización del entorno, 2) Organización de la tarea, 3) Búsqueda de información y 4) Hábitos inadecuados de regulación. Respecto a las propiedades psicométricas del SRSI-SR adaptado, éste mostró adecuadas propiedades psicométricas en una población de estudiantes regulares de un programa de pregrado en Psicología de Colombia. Este instrumento destaca por su sencillez y rápida administración. No obstante, los autores del SRSI-SR adaptado no realizaron un procedimiento de análisis factorial confirmatorio (AFC) para confirmar la estructura del instrumento ya que un AFC permite evaluar la validez y fiabilidad de cada ítem (Batista-Foguet et. al., 2004; Streiner et. al, 2015). Por ello, en esta investigación se busca evaluar dicho instrumento a través de la exploración de sus propiedades psicométricas usando análisis factorial exploratorio (AFE) y análisis factorial confirmatorio (AFC). 
Por consiguiente, el instrumento SRSI-SR adaptado puede ser útil en la distinción de estudiantes universitarios que tienen hábitos inadecuados de regulación versus estudiantes que utilizan estrategias de autorregulación como organización de la tarea, organización del entorno y búsqueda de información. Adicionalmente, es importante mencionar que el instrumento SRSI-SR adaptado se asocia al modelo de tres etapas de Zimmerman de acuerdo con lo expuesto por Hernández y Camargo (2017) quienes indican que: "a partir de las conceptualizaciones planteadas para los factores del SRSI-SR adaptado, se puede conjeturar que los factores están ubicados diferencialmente en el proceso cíclico de tres etapas de ARA (Zimmerman, 2011): previsión, ejecución y autorreflexión" (Hernández y Camargo, 2017, p.15). Finalmente, para que este instrumento sirva al propósito, debe reunir los atributos y características psicométricas exigibles a un instrumento. Por esta razón la investigación busca responder a la siguiente pregunta ¿Tendrá el SRSI-SR adaptado las características psicométricas adecuadas para ser considerado un instrumento válido y fiable en estudiantes universitarios de las carreras de pedagogía? En concordancia con la pregunta de investigación el objetivo del estudio es validar la escala SRSI-SR adaptado en población de estudiantes de pedagogía chilenos usando la versión en español desarrollada por Hernández y Camargo (2017a). Para dar respuesta al objetivo y pregunta de investigación se lleva a cabo un estudio instrumental (Ato. et. al, 2013) que incluye el análisis de las propiedades psicométricas del SRSI-SR adaptado a través de un análisis factorial exploratorio (AFE) y análisis factorial confirmatorio (AFC). En consecuencia, la investigación busca aportar con un instrumento a la actividad académica que puede resultar muy útil tanto en la formación de profesores, como en la actividad de orientación psicopedagógica a los estudiantes de primer año de universidad.

\section{MÉTODO}

En esta investigación se utiliza un enfoque cuantitativo que se clasifica como un estudio instrumental (Ato. et. al, 2013). A continuación, se describe a los participantes, instrumentos y procedimiento de la investigación.

\section{Participantes}

Para la selección de los participantes se consideró como técnica el muestreo no probabilístico debido a que se escogió de forma intencionada estudiantes que cursaran primer año de la carrera de pedagogía de una universidad de Chile. Se encuestó a 382 estudiantes, con participación voluntaria, pertenecientes a siete especialidades. El $37,89 \%$ de los participantes son hombres y el $62.11 \%$ son mujeres. La edad media es de 19.17 años.

\section{Instrumento}

Se utilizó la escala SRSI-SR adaptado para estudiantes universitarios por Hernández y Camargo (2017a) porque la versión SRSI-SR original es para estudiantes de enseñanza secundaria (Cleary, 2006). El instrumento SRSI-SR adaptado está compuesto por 18 ítems agrupados en 4 factores que se definen conceptualmente como:

(1) Factor Organización del Entorno (OE): se conceptualiza como las acciones anticipadas o realizadas por el individuo para disminuir las variables distractoras, que comprometen la definición y ocupación de un sitio de estudio, y la priorización de las actividades académicas respecto a actividades no académicas.

(2) Factor Búsqueda de Información (BI): se define como el conjunto de acciones deliberadas orientadas a la identificación y uso de fuentes de información necesarias para el desarrollo de una actividad académica

(3) Factor Organización de la Tarea (OT): se concibe como la planeación de la secuencia de acciones requeridas para la realización de una actividad académica a partir de la precisión de un estado meta y la identificación de los recursos disponibles.

(4) Factor Hábitos Inadecuados de Regulación (HIR): es definido como el conjunto de conductas evitativas del individuo respecto al cuidado e involucramiento activo en actividades de aprendizaje académico.

\section{Procedimiento}

EI SRSI-SR adaptado, posee ítems politómicos (tipo Likert), los que originalmente tenían cuatro valoraciones posibles (nunca, casi nunca, casi siempre, siempre). Sobre la base de que un número de opciones de respuesta de 5 o más contribuye a que las distribuciones puedan alcanzar la normalidad aproximada, el instrumento se modificó añadiendo una opción adicional "a veces" al centro de valoración (Streiner et.al., 2015). Un motivo empírico para agregar una valoración más en la escala Likert se observó en la aplicación del piloto donde se detectó problemas en los estudiantes para responder a los ítems usando solo las cuatro valoraciones. La administración piloto, mediante una entrevista cognitiva (Smith-Castro y Molina, 2011), se realizó a un grupo de 10 estudiantes universitarios de distintos niveles, carreras y rendimiento heterogéneo con el propósito de verificar la comprensión de cada uno de los ítems. En los resultados de esta aplicación piloto no se detectó ningún problema de comprensión de los ítems. Lo que significa que el contenido de los 
ítems no interfiere con la respuesta a los ítems, ni en la aplicación piloto, ni en la aplicación final. Luego, con el objetivo de determinar el tiempo requerido para la aplicación, se realizó otro piloto a 5 estudiantes. Por último, la aplicación final se llevó a cabo durante el primer y segundo semestre del año académico 2017 en laboratorios de computación. Los estudiantes contestaron de forma voluntaria y con consentimiento informado el instrumento en grupos de no más de 25 personas en sesiones de menos de 20 minutos.

\section{ANALISIS Y PROPIEDADES PSICOMETRICAS DEL INSTRUMENTO}

En este apartado se presenta el análisis de las propiedades psicométricas de la escala SRSI-SR adaptado con el propósito de dar cumplimiento al objetivo de esta investigación. El análisis se realiza en el marco de la teoría clásica de los test Muñiz (2010).

\section{Proceso de análisis}

La primera actividad que se realizo fue retirar los valores extremos a través del análisis del comportamiento de ítems, que es necesario en el proceso de validación de un instrumento. Después, se aplicó un AFE considerando factores asociados a los autovalores mayores que uno. Debido a la cantidad de muestras, se usó el método de extracción de mínimos cuadrados no ponderados (ULS, por sus siglas en inglés), considerando una rotación oblicua mediante Promax (Kappa=4) por considerar que conceptualmente los factores guardan relación entre ellos (Costello y Osborne, 2011; Streiner et.al.,2015). Además, se verificó la relación parcial entre los ítems a través de la medida Kaiser-Meyer-Olkin (KMO), considerando como criterio a valores $\mathrm{KMO}>0.8$ como buenos. Tras la rotación, se descartaron los ítems de la matriz rotada que no tuviesen una contribución mínima de .40 (Ferrando y Lorenzo-Seva, 2014; Lloret-Segura et.al.,2014; Marsh, 2014). Para la validación interna de cada factor se calculó el Alpha de Cronbach, considerando valores $\alpha>.80$ como fiables (Hu y Bentler, 1999).

Luego, de definir los ítems asociados a cada factor sugerido por el AFE, se llevó a cabo un AFC utilizando un algoritmo de ajuste por máxima verosimilitud. Las medidas de ajuste del modelo fueron obtenidas para tres modelos: 1) utilizando los cuatro factores y los 18 ítems tal cual son presentados en Hernández y Camargo (2017a), el cual se denomina en adelante "modelo original"; 2) la versión sugerida por el AFE, llamado "modelo exploratorio"; 3) la versión optimizada según los índices que es llamado "modelo confirmatorio". Para evaluar la bondad de ajuste de los modelos, se utilizaron índices de ajuste absolutos (SRMR, GFI, AGFI), un indicador corregido por parsimonia (RMSEA) e índices comparativos incrementales (NFI, NNFI, TLI, CFI, IFI) (BatistaFoguet et.al,2004; Brown, 2014). Estos índices y la definición de los criterios usados para valorar los índices como buenos o malos son presentados en la Tabla 1 (Streiner et.al., 2015). En ésta, los intervalos implícitamente definidos entre Bueno y Malo corresponden a valoraciones consideradas 'Adecuadas'.

Tabla 1. Criterios para la valoración de los índices de bondad de ajuste.

\begin{tabular}{|c|c|c|c|c|}
\hline Valoración & SRMR & GFI & AGFI & RMSEA \\
\hline Bueno & $<.05$ & $>.90$ & $>.90$ & $<.05$ \\
Adecuado & entre $.05 \mathrm{y} .08$ & entre $.80 \mathrm{y} .90$ & entre $.50 \mathrm{y} .80$ & entre $.05 \mathrm{y} .08$ \\
Malo & $>.08$ & $<.80$ & $<.80$ & $>.08$ \\
\hline
\end{tabular}

\section{Análisis Factorial Exploratorio (AFE)}

En primera instancia se identificaron los ítems con valoración invertida, ítems 1 y 9 , transformándolos acorde a la escala definida. Los 18 ítems del instrumento fueron sometidos a un AFE considerando los factores con autovalores mayores a 1. Los resultados del proceso mostraron la existencia de 4 factores que cumplían este criterio, esto concuerda con los cuatro factores definidos en el instrumento SRSI-SR adaptado en Colombia por Hernández y Camargo (2017a). Esta configuración de factores e ítems se denominó "modelo original", cuya matriz de factores rotada es presentada en la Tabla 2. La medida de adecuación $\mathrm{KMO}=.883(\mathrm{p}<.001)$, muestra que la reducción de factores realizada es viable mediante el método utilizado, alcanzando una varianza explicada acumulada de $55.939 \%$.

Con el resultado obtenido, se descartaron los ítems que en la matriz rotada no tuvieron las contribuciones suficientes (< 0.40) según criterios AFE (Ferrando y Lorenzo-Seva, 2014; Lloret-Segura et.al.,2014; Baglin, 2017). Esto último, derivó en el descarte de 2 ítems por baja contribución que son; ítem $5=-.302$ e ítem $18=0.302$ y 0.309 . En efecto, el ítem 18 contribuye a dos factores diferentes. Al modelo sin los ítems 5 y 18 se le denominó "Modelo exploratorio". También, en la Tabla 2 se observa que el ítem 1 del factor HIR (originalmente) contribuye al factor BI, mientras que el ítem 9 del factor OE (originalmente) contribuye al factor HIR. De esta forma, para realizar un análisis comparativo, el "Modelo Original", el "Modelo Exploratorio" y un 
tercer modelo llamado "Modelo Confirmatorio", que incluye el cambio de factor para los ítems 1 y 9 a los factores $\mathrm{BI}$ y HIR respectivamente fueron sometidos a un análisis factorial confirmatorio.

Tabla 2. Ponderación ítems Análisis Factorial Exploratorio (AFE)

\begin{tabular}{|c|c|c|c|c|}
\hline \multirow{2}{*}{ Ítems y Factores } & \multicolumn{4}{|c|}{ Factor } \\
\hline & 1 & 2 & 3 & 4 \\
\hline Hábitos inadecuados de regulación (HIR) & $(O T)$ & $(H I R)$ & $(B I)$ & $(O E)$ \\
\hline 1.Cuando no comprendo algún tema le pregunto al profesor & & & .474 & \\
\hline 2.Evito preguntar en clase cuando no entiendo el tema & & .693 & & \\
\hline 3.Me rindo fácilmente cuando no entiendo algo & & .889 & & \\
\hline 4.Cuando estoy estudiando ignoro los temas que son difíciles de entender & & .809 & & \\
\hline 5. Me distraigo fácilmente cuando estoy estudiando & -.302 & & & \\
\hline \multicolumn{5}{|l|}{ Organización del entorno (OE) } \\
\hline 6. Intento estudiar en un sitio tranquilo & & & & .718 \\
\hline $\begin{array}{l}\text { 7.Intento estudiar en un lugar sin distracciones (ruido, gente } \\
\text { hablando) }\end{array}$ & & & & .907 \\
\hline $\begin{array}{l}\text { 8.Me aseguro de que nadie me distraiga cuando estoy } \\
\text { Estudiando }\end{array}$ & & & & .636 \\
\hline $\begin{array}{l}\text { 9.Permito que las personas me interrumpan cuando estoy } \\
\text { Estudiando }\end{array}$ & & .457 & & \\
\hline $\begin{array}{l}\text { 10.Termino todas mis actividades académicas antes de iniciar } \\
\text { otro tipo de actividades }\end{array}$ & & & & .484 \\
\hline \multicolumn{5}{|l|}{ Búsqueda de información (BI) } \\
\hline $\begin{array}{l}\text { 11. Realizo búsquedas bibliográficas adicionales que me ayuden a } \\
\text { comprender los temas de clase }\end{array}$ & & & .927 & \\
\hline 12.Busco material complementario de los temas vistos en clase & & & .823 & \\
\hline $\begin{array}{l}\text { 13. Investigo cuando no entiendo algo sobre las tareas que me } \\
\text { Dejan }\end{array}$ & & & .426 & \\
\hline \multicolumn{5}{|l|}{ Organización de la tarea (OT) } \\
\hline 14.Planeo en qué orden realizaré mis actividades académicas & .724 & & & \\
\hline 15.Coordino mi tiempo de acuerdo a las actividades académicas asignadas & .809 & & & \\
\hline 16. Hago un horario para organizar mi tiempo de estudio & .953 & & & \\
\hline $\begin{array}{l}\text { 17.Uso algún método para mantener en orden el material de } \\
\text { mis clases }\end{array}$ & .757 & & & \\
\hline $\begin{array}{l}\text { 18.Antes de empezar a estudiar, pienso cuál es la mejor forma } \\
\text { de hacerlo }\end{array}$ & .302 & .309 & & \\
\hline Porcentaje de varianza explicada acumulada & 34.2 & 45.3 & 51.6 & 55,9 \\
\hline
\end{tabular}

\section{Análisis Factorial Confirmatorio (AFC)}

En esta etapa los tres modelos fueron evaluados mediante AFC. Así, los resultados obtenidos del AFC para el "modelo confirmatorio", ratificaron que los ítems 5 y 18 covarían con otros factores, por lo que se optó por eliminarlos siguiendo los lineamientos de un AFC cuya ventaja es que permiten evaluar la validez y fiabilidad de cada ítem (Batista-Foguet et.al,2004; Brown, 2014; Streiner et.al.,2015). Así, se generó el "Modelo Final" que se muestra en la Fig.1. De acuerdo con lo señalado anteriormente, más los índices de bondad de ajuste de cada modelo presentados en la Tabla 3 y considerando los criterios de evaluación explicitados anteriormente para cada índice, el "Modelo Confirmatorio" (Modelo Final) tiene un ajuste "adecuado" para los índices RMSEA y SRMR, un ajuste "bueno" para los índices GFI, AGFI y el mejor ajuste para los índices comparativos NFI, IFI, CFI y AIC. Cabe señalar que el análisis AFC se realizó en el software EQS porque permite usar modelos robustos. (Por requerimientos de la revista, el modelo originado en EQS se transcribió a word). 
Tabla 3. Índices de ajuste para cada modelo

\begin{tabular}{|l|c|c|c|c|c|c|c|c|}
\hline \multicolumn{1}{|c|}{ Modelo } & RMSEA & SRMR & GFI & AGFI & NFI & IFI & CFI & AIC \\
\hline Modelo Original (18 ítems) & 0.116 & .1327 & .802 & .738 & .771 & .807 & .805 & 753.300 \\
Modelo Exploratorio (16 ítems) & 0.108 & .0956 & .857 & .796 & .846 & .875 & .873 & 463.341 \\
Modelo Confirmatorio (16 ítems) & .0710 & .0704 & .930 & .891 & .910 & .939 & .938 & 236.631 \\
\hline
\end{tabular}

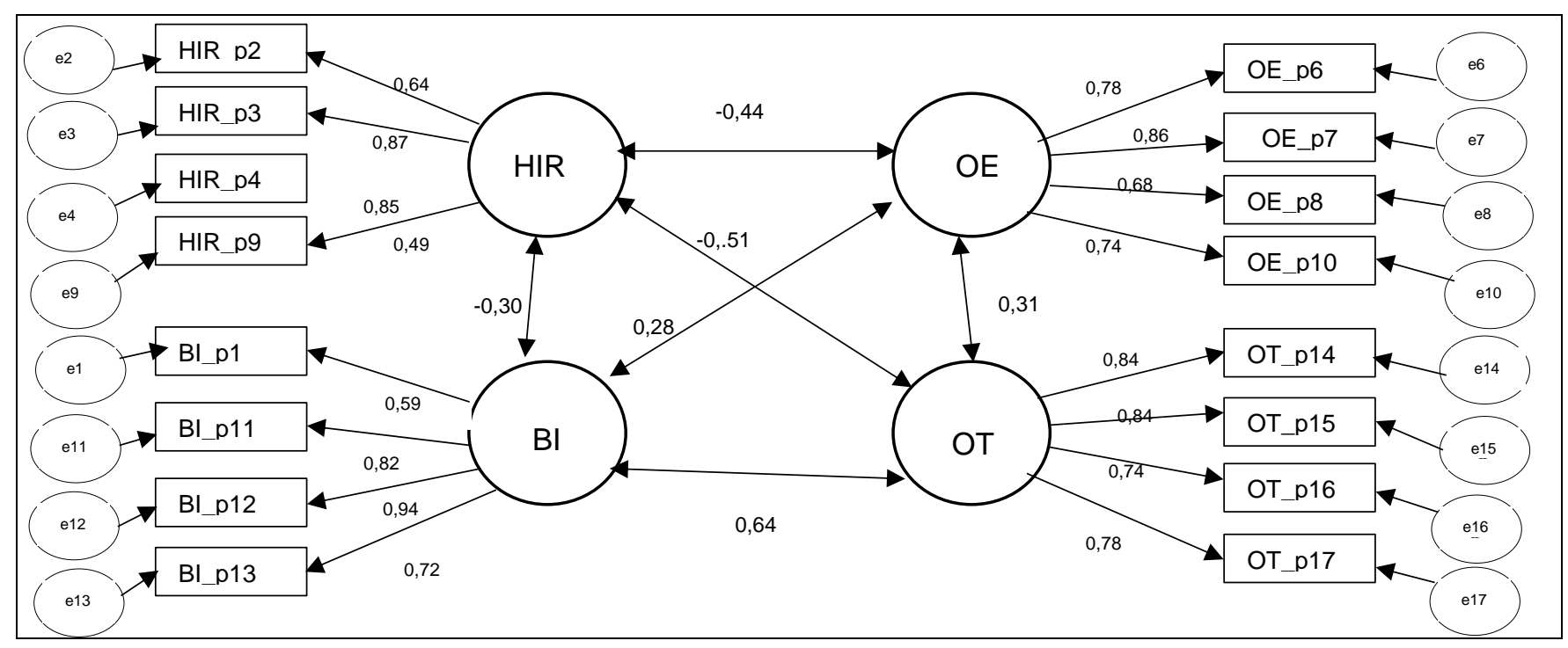

Fig. 1. Modelo Final aplicado el Análisis Factorial Confirmatorio.

En la Fig.1., se observa el modelo final producto de la aplicación del AFC. Los círculos (centrales) correlacionados entre sí representan los factores, y los rectángulos a los ítems. El factor HIR se correlaciona inversamente con los factores OE, OT y BI.

\section{DISCUSIÓN}

El propósito del estudio fue analizar las propiedades psicométricas de la escala SRSI-SR adaptado en Colombia por Hernández y Camargo (2017a) mediante un análisis factorial exploratorio y confirmatorio para el contexto de educación superior en una muestra de estudiantes chilenos universitarios de siete careras de pedagogía. En este contexto, los resultados asociados a las propiedades psicométricas del instrumento muestran un adecuado funcionamiento de la escala SRSI-SR adaptado, lo que posibilita su aplicación en población de estudiantes de pedagogía en chile. Se evidencio una estructura factorial, que da cuenta de 4 factores: 1) Organización del entorno, 2) Organización de la tarea, 3) Búsqueda de información y 4) Hábitos inadecuados de regulación. Estos cuatro factores coinciden con los factores del instrumento adaptado en Colombia. Además, el "Modelo Confirmatorio" resultante del AFC incluye el cambio de factor para los ítems 1 y 9 a los factores BI y HIR respectivamente.

La consistencia interna del instrumento global es alta $(\alpha=.89)$ de acuerdo con Hu y Bentler (1999). Respecto a la cantidad de ítems de los factores, estos mantienen el número de ítems para considerar la constitución de un factor (Costello y Osborne, 2011). Por los antecedentes anteriores, se comprueba que el instrumento mide los cuatro factores reportados por Hernández y Camargo (2017a) y que las ventajas del instrumento validado son sencillez y facilidad de administración lo que posibilitará su utilización en nuevos estudios. Sin embargo, se debe mencionar que la escala SRSI-SR adaptado difiere de la escala original creada por Cleary (2006) pues la escala de este autor consta de tres dimensiones y la escala de Hernández y Camargo (2017a), que se examinó psicométricamente en esta investigación tiene 4 factores. En suma, el análisis psicométrico realizado en este estudio aporta al enriquecimiento de los datos de aplicación del instrumento SRSI-SR adaptado, es decir, a los datos de comportamiento del inventario en una nueva población.

En relación con estudios sobre estrategias de autorregulación, se debe mencionar que en Latinoamérica existen pocas investigaciones que midan esta variable. Esta situación se evidencia en la revisión sistemática realizada por Hernández y Camargo (2017b) quienes reportan que entre 2005 y 2015 se publicaron 7 artículos donde se incluye como participantes a estudiantes de pedagogía (Hernández-Pina, Rosario y Cuesta Sáez, 2010; Simão y Flores, 2010; De la Fuente et al., 2012; Pranke, Garello y Rinaudo,2013; Paoloni y VajaArabela, 2013; Marini y Boruchovitch, 2014; Bragagnolo-Frison, 2015). Esta evidencia empírica respecto a la 
baja cantidad de estudios en Latinoamérica que incluyan la participación de estudiantes de pedagogía sugiere que se debe impulsar la investigación relacionada con ARA en los profesores en formación pues ellos podrían influir en la autorregulación de sus estudiantes mediante el uso de estrategias que fomenten ARA en el aula (Peeters et. al., 2014; Zambrano, 2016; Daura, 2017, entre otros). En este sentido, la validación del instrumento SRSI-SR adaptado contribuye para que sea aplicado en futuros estudios en esta población. Así, se podría seguir impulsando la investigación con datos empíricos en Latinoamérica.

Las limitaciones de la investigación se relacionan con el tipo de muestra utilizada, pues participaron sólo estudiantes de distintas carreras de pedagogía que pertenecen a una misma institución universitaria. En futuras investigaciones para confirmar la validez y confiabilidad de la escala en estudiantes universitarios chilenos, se ampliará la muestra con estudiantes del área ingeniería y área de la salud. No obstante, los resultados de las propiedades psicométricas preliminares para la utilización de la escala SRSI-SR adaptado en estudiantes de pedagogía chilenos significa el aporte de un instrumento a la actividad académica en el ámbito de utilización para el área de estudiantes de pedagogía. Por ello, su uso puede resultar muy útil tanto en la formación de profesores, como en la actividad de orientación psicopedagógica a los estudiantes de primer año de universidad. Como proyección futura se podría realizar un estudio transcultural y un estudio longitudinal.

\section{CONCLUSIONES}

De acuerdo con el objetivo planteado en este estudio y en base a los resultados asociados a las propiedades psicométricas del instrumento se concluye: 1) Adecuado funcionamiento de la escala SRSI-SR adaptado, lo que posibilita su aplicación en población de estudiantes de pedagogía chilenos. 2) La estructura factorial da cuenta de 4 factores que coinciden con los factores del instrumento adaptado en Colombia. En consecuencia, el instrumento es válido y fiable para ser utilizado en esta población de estudiantes, aportando a la comunidad académica que desee realizar estudios para medir la variable estrategias de autorregulación usando un instrumento sencillo y de fácil administración.

\section{AGRADECIMIENTOS}

Este artículo se realizó en el contexto del Proyecto FONDECYT 1161502 Modelo explicativo de la permanencia y el abandono de los estudios universitarios, basado en procesos cognitivos y motivacionales.

\section{REFERENCIAS}

Ato, M., J. López y A. Benavente, Un sistema de clasificación de los diseños de investigación en psicología, doi:10.6018/analesps.29.3.178511, Anales de Psicología, 29(3), 1038-1059 (2013)

Baglin, J., Improving your exploratory factor analysis for ordinal data: A demostration using FACTOR, Practical Assessment, Research \& Evaluation, 19, 2-11 (2014)

Batista-Foguet, J. M., G. Coenders y J. Alonso, Análisis factorial confirmatorio. Su utilidad en la validación de cuestionarios relacionados con la salud, Medicina Clínica, 122(1), 21-27 (2004)

Boekaerts, M., Self-regulated learning: Bridging the gap between metacognitive and metamotivation theories, doi:10.1207/s15326985ep30044, Educational Psychologist, 30(4), 195-200 (1995)

Boekaerts, M., P.R. Pintrich y M. Zeidner, Handbook of self-regulation, 2a Ed., 417-451, Elsevier Academic Press, San Diego, CA (2005)

Broadbent, J., y W. Poon, Self-regulated learning strategies \& academic achievement in online higher education learning environments: A systematic review, doi:10.1016/j.iheduc.2015.04.007, The Internet and Higher Education, 27, 1-13 (2015)

Brown, T.A., Confirmatory factor analysis for applied research, 1ํㅡㄹ. Ed., 35-55, Guilford, NY, USA (2014)

Cazan, A.M., Self-regulated learning strategies-predictors of academic adjustment, doi: 10.1016/jsbspro.2012.01.092, Procedia-Social and Behavioral Sciences, 33, 104-108 (2012)

Cleary, T.J., The development and validation of the self-regulation strategy inventory-self-report, doi: 10.1016/j.jsp.2006.05.002, Journal of School Psychology, 44(4), 307-322 (2006)

Cleary, T.J. y P. Platten, Examining the correspondence between self-regulated learning and academic achievement: $\mathrm{A}$ case study analysis, doi: 10.1155/2013/272560, Education Research International, 18, 1-18 (2013)

Costello, A.B. y J.W Osborne, Best practices in exploratory factor analysis: Four recommendations for getting the most from your analysis, doi:10.1.1.110.9154, Practical Assessment Research Evaluation, 10(7), 1-8 (2011)

Daura, F. T., Aprendizaje autorregulado e intervenciones docentes en la universidad, doi: 10.15517/revedu.v41i2.21396, Revista Educación, 41(2), 1-19 (2017)

Díaz, A. y M.V. Pérez, Autoeficacia, enfoque de aprendizaje profundo y estrategias de aprendizaje, doi: 10.17060/ijodaep, Inter. Journal of Developmental and Educational Psychology: INFAD. Revista de Psicología, 2(1), 341-346 (2013) 
Ferrando, P. J. y U. Lorenzo-Seva, El análisis factorial exploratorio de los ítems: algunas consideraciones adicionales, doi:10.6018/analesps.30.3.199991, Anales de psicología, 30(3), 1170-1175 (2014)

Garello, M. V. y M.C Rinaudo, Autorregulación del aprendizaje, feedback y transferencia de conocimiento: Investigación de diseño con estudiantes universitarios, Revista electrónica de investigación educativa, 15(2), 131-147 (2013)

Gutiérrez-Braojos, C., H. Salmerón-Pérez y J. Muñoz-Cantero, El efecto modulador los patrones temporales sobre el logro en el aprendizaje autorregulado, doi:10.1387/RevPsicodidact.10066, Psicodidáctica, 19(2), 267-287 (2014)

Hernández, A. y A. Camargo, Adaptation and validation of Self-Regulation Strategy Inventory-Self-Report in university students, doi:10.1016/j.sumpsi.2017.02.001, Suma Psicológica, 24(1), 9-16 (2017a)

Hernández, A. y A. Camargo, Aprendizaje Autorregulado en la educación superior en Iberoamérica: una revisión sistemática, doi: 10.1016/j.rlp.2017.01.001, Revista Latinoamericana de Psicología, 49(2), 146-160 (2017b)

Hernández-Pina, F., P. Rosário y J.D. Cuesta Sáez de Tejada, Impacto de un programa de Aprendizaje Autorregulado en estudiantes de Grado, doi:10.4438/1988-592X-0034-8082-RE, Revista de Educación, 353, 571-588 (2010)

$\mathrm{Hu}$, L. y P. Bentler, Cutoff criteria for fit indexes in covariance structure analysis: Conventional criteria versus new alternatives Structural Equation Modeling, doi:10.1080/10705519909540118, A Multidisciplinar Journal, 6(1), 1-55 (1999)

Lloret-Segura, S., A. Ferreres-Traver y otros dos autores, El análisis factorial exploratorio de los ítems: una guía práctica, revisada y actualizada, doi:10.6018/analesps.30.3.199361, Anales de Psicología, 30(3), 1151-1169 (2014)

Marini, J.A.D.S. y E. Boruchovitch, Self-Regulated Learning in Students of Pedagogy, doi:10.1590/198243272459201406, Paidéia (Ribeirão Preto), 24(59), 323-330 (2014)

Marsh, H. W., A. J. Morin y otros dos autores. Exploratory structural equation modeling: An integration of the best features of exploratory and confirmatory factor analysis, doi: 10.1146/annurev-clinpsy-032813-153700, Annual review of clinical psychology, 10, 85-110 (2014)

Mega, C., L. Ronconi y R. de Beni, What makes a good student? How emotions, self-regulated learning, and motivation contribute to academic achievement, doi:10.1037/a0033546, Journal of Educational Psychology, 106(1), 121-131 (2014)

Muñiz, J., Las Teorías de los tests: teoría clásica y teoría de respuesta a los ítems. Papeles del Psicólogo, 31(1), 57-66 (2010)

Peeters, J., F. De Backer y otros cuatro autores, The role of teachers' self-regulatory capacities in the implementation of self-regulated learning practices, doi: 10.1016/j.sbspro.2014.01.504, Procedia - Social and Behavioral Sciences, 116, 1963-1970 (2014)

Pintrich, P. y E.V De Groot, Motivational and Self-Regulated Learning Components of Classroom Academic Performance, doi:10.1.1.315.6253, Journal of Educational Psychology, 82(1), 33-40 (1990)

Pintrich, P., D. Smith y otros dos autores. A Manual for the Use of the Motivated Strategies for Learning Questionnaire (MSLQ), 1를 Ed., Michigan, University of Michigan (1991)

Pranke, A. y L. M Bragagnolo Frison, Potencialização da Aprendizagem Autorregulada de Bolsistas do PIBID/UFP el do curso de Licenciatura em Matemática a través de Oficinas Pedagógicas, doi:10.1590/1980-4415v29n51a12, Boletim de Educação Matemática, 29(51) (2015)

Salmerón, H., C. Gutierrez-Braojos y otros dos autores, Metas de logro, estrategias de regulación y rendimiento académico en diferentes estudios universitarios, Revista de Investigación Educativa, 467-486 (2011)

Sistema de Información de la Educación Superior, SIES, Informe Retención de 1er año de pregrado, cohortes 2011-2015, Santiago de Chile (2016)

Smith-Castro, V. y M. Molina, Cuaderno Metodológico 5. La entrevista cognitiva: guía para su aplicación en la evaluación y mejoramiento de instrumentos de papel y lápiz. San José, CR.: Instituto de Investigaciones Psicológicas, Universidad de Costa Rica (2011)

Streiner, D. L., G.R Norman y J. Cairney, Health measurement scales: a practical guide to their development and use, $5^{\mathbf{a}}$ Ed., 7-17, Oxford University Press, NY, USA (2015)

Winne, P. H. A metacognitive view of individual differences in self-regulated learning, doi:10.1016/S1041-6080(96)900229, Learning and Individual Differences, 8(4), 327-353 (1996)

Zambrano, C., Autoeficacia, Prácticas de Aprendizaje Autorregulado y Docencia para fomentar el Aprendizaje Autorregulado en un Curso de Ingeniería de Software, doi:10.4067/S0718-50062016000300007, Formación Universitaria, 9(3), 51-60, (2016)

Zimmerman, B., Handbook of self-regulation, $1^{\text {a }}$ Ed., 13-40, Academic Press, San Diego, California (2000) 